\title{
ALGORITHMS FOR COMPUTING OPTIMAL POLICIES IN QUEUEING NETWORKS WITH PARTIAL INFORMATION
}

\section{J.A. Loeve, University of Leiden, NL}

In a Markov decision chain the optimal policy can be found by successive approximation. However, if it is not possible to observe all states of the system, the policy found in this way is not useful in practice. This is one of the reasons for looking at Markov decision chains with partial state information.

For example, this is the case in decentralized queueing networks. In such a queueing network there are several nodes or centres and each centre has its own decisionmaker who controls that centre. Each decisionmaker can observe only one centre, so the decisions are based on the information about that particular centre.

In general we can model this kind of situation by making a partition of the state space. Now the information on the state of the system available on each decision moment is the set of the partition in which the state is contained. So the exact state is not known and the decisions in all states in a set of the partition have to be the same.

Several new algorithms to obtain optimal policies in Markov decision chains with partial state information are designed. They can handle a larger state space than the well-known algorithms. However, there is no proof of convergence yet, although in practice it seems to work quite well. We will analyse these algorithms and discuss their applicability for finding decentralized optimal policies in some specific queueing models. 